\title{
SIRIUS - Saarbrücken Information Retrieval and Interchange Utility Set
}

\author{
Reiner GÜTTLER and Ralf DENZER \\ Hochschule für Technik und Wirtschaft des Saarlandes \\ Goebenstr. 40, D-66117 Saarbrücken, Germany, tel. 49-681-5867-238 or 426, \\ fax 49-681-5867-122, email guettler@htw.uni-sb.de or denzer@htw.uni-sb.de
}

\begin{abstract}
The SIRIUS-project deals with the development of a prototype of a tool set for the integration of heterogenous subsystems fournishing environmental information. We first discuss the major integration problems which delay the development of Environmental Information Systems (EIS). The objectives of the SIRIUS-project and its integration concept are presented. Finally we describe the implementation of the prototype and its functionalities.
\end{abstract}

\section{Keywords}

Environmental Information Systems (EIS), integration, open distributed systems, meta-information

\section{$1 \quad$ PROBLEMS IN THE FIELD OF EIS}

Since the midth of the 80th we observe an increasing use of computer based methods in dealing with environmental problems. There are two main reasons :

an increasing pressure on environmental authorities by the public ending in additional laws and an increasing state of knowledge in the field. Both reasons lead to an enormously increasing amount and complexity of work for those authorities which they can no longer overcome without computer based methods.

Typical aspects of environmental computer applications are often the interdisciplinarity and the need for data and methods coming from different sources. This leads to the main problem of integration of environmental data and methods into an Environmental Information System (EIS).

One approach could be a centralized solution with a top-down designed overall system to avoid heterogenous subsystems. Many disadvantages of such an approach have soon become obvious, among others :

specific objectives of different subsystems need different hard-/softwaresolutions

already existing subsystems cannot be integrated 
We state that it is impossible for overall systems of such a size

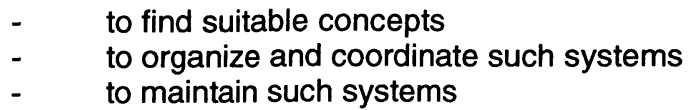

under the condition that every user has a solution which fits his specific needs.

This leads to the alternative idea of a completely decentralized open distributed EIS, in which we "can live with heterogenous subsystems". Subsystems can be heterogenous in different aspects - syntax, semantics and structure - which we will explain shortly.

Heterogenous syntax means that the subsystems can differ in hardware, operating system, data base, local network, communication and user-interface software. Since the typical users (e.g. in authorities) in general will not have a high qualification in dealing with computers, heterogenous syntax of subsystems must be hidden for those users. With the increasing efficiency of modern software-tools this kind of heterogeneity should no longer constitute an obstacle too difficult to overcome.

Heterogenous semantics seems much more difficult in our opinion. We understand by heterogenous semantics different comprehension of the meaning of an information. This starts by differing meanings of a single information (e.g. different names for the same object, the same name for different objects) up to different association of additional information to a specific object. There are many aspects of heterogenous semantics (see Denzer,1994a for a detailed explanation).

Heterogenous structure means the use of different data structures for the same objects in different subsystems. Since already the raw data can be heterogenous in syntax and semantics, the structures will be heterogenous by this fact, but a new type of heterogeneity arises by the different aggregations and components of raw data to built structures.

By the use of meta-data the problems arising from the different kinds of heterogeneity can be documented but in general not be removed. We can for example state by the use of meta-data that two pieces of information that we want to compare are not comparable because of different measurement methods, different aggregations and so on. There are only few cases, where computer based methods can help in this situation, for example by conversion of data following a standard framework. The reason for the problem lies much deeper. The different scientific disciplines in the field (biologie, chemistry and so on) should be aware, that computer based methods in general cannot heal a lack of strategie in the production of raw data.

The dynamic behaviour of the different subsystems constitutes another difficult problem for integration. The scientific methods in the field of ecology are still young. So it is inevitable that the subsystems must be able to develop independently with the increasing knowledge in the field. 
Still another problem arises from the autonomy of the different authorities that are often responsable for the different subsystems. In many cases an institution (e.g. a ministry) that needs data from a subsystem does not have the right to give orders to the institution responsable for the subsystem (depending from another ministry).

All those problems constitute at once arguments against a centralized approach but also quite difficult obstacles for a distributed solution. They are described in more detail in GÜTTLER, 1993, DENZER, 1994a and DENZER, 1995.

\section{OBJECTIVES OF THE SIRIUS-PROJECT}

The main objectives of the SIRIUS-project were the conception and a prototype for an open distributed system combining different heterogenous subsystems including the necessary meta-informations.

The solution we were looking for should satisfy the following requirements :

a) The solution should allow to integrate subsystems which are heterogenous in any aspect (syntax, semantics, structure). This means that the system can work without any structure of meta-data or raw-data imposed by any organisation. Obviously it is possible for the system to work with an imposed structure by a ministry for example. But it is important that the system is not the restricting factor. So the system can adapt to the problem, e.g. organisation needs, and not viceversa.

b) Any client should be able to investigate any subsystem for meta-data and the raw-data behind with one single tool. It should also be possible to transport the needed data through the network.

c) The solution should allow any subsystem to modify dynamically. This means that neither any client neither the investigation tool need to be modified, if a subsystem changes its structure. Next time a client accesses the modified subsystem he will notice the modification.

\section{THE INTEGRATION CONCEPT}

The requirements lead to some concepts based on ideas of several earlier projects (DENZER, 1993, SCHIMAK, 1994). From DENZER, 1993 comes a two-step model for an open EIS satisfying point a) and b) of the requirements. The model tries to seperate the components of the local systems from the network by a kind of abstraction realized by a layer between local systems and network, the components of this layer beeing some public services. The local systems treat their data using local conventions and following local needs. They are designed for local purposes and very often exist already when the problem arises to let them communicate through a network. This means that the design of the local systems very often did not take into account the fact to open the system for external access. The local systems have an interface with the components of the intermediate layer (S1), while these components communicate via a standard protocol (SO) through the network. 


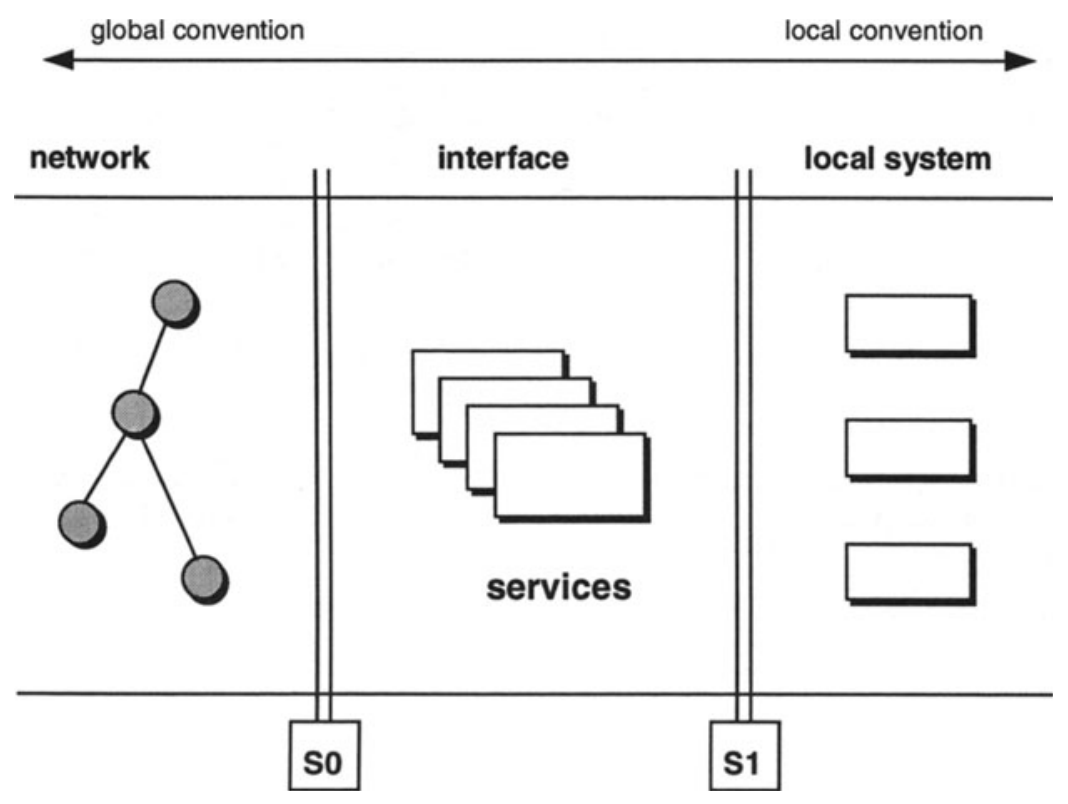

A local object interface (S1) is used for the local communication of the subsystems with the services available on every node of the network. A general object interface (SO) is used for the communication of the different servers. This general object interface must be publicly agreed upon.

A successfull implementation of the basic idea of this concept is described in SCHIMAK, 1994. But this implementation does not yet satisfy the requirement C). A client must know the semantics and structure of an object fournished by any server.

But based on experiences in the above cited implementation an additional idea (see also DENZER, 1994b) was integrated in the SIRIUS-concept : the abstract object interface. It means that any subsystem should be able to describe its content over an interface which is uniform in the whole system. The interface must be able to communicate any information types with their relations. Since every information communicates in addition its structure the clients are not sensible for modifications and thus the requirement $\mathrm{c}$ ) is satisfied.

Example : it is possible to implement an Eenvironment Data Catalogue (EDC) visualization-tool in such a way, that a client must not be modified even if

- $\quad$ the structure of the EDC is modified dynamically

- $\quad$ object classes in the EDC are modified dynamically

- $\quad$ at different nodes are different EDC's 
We give first a list of the SIRIUS components and describe below how these components can work together to match our concepts.

The main SIRIUS-components are:

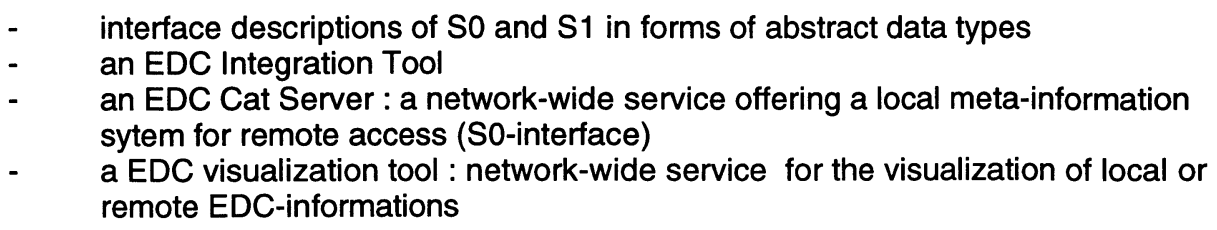

Besides these main components there are some C-programmed interfaces helping to use local tools to access the communication interfaces or to use SIRIUS userinterface-objects for local applications.

Following the SIRIUS-concepts the implementation allows the different nodes to access the SIRIUS-system as an information server at different levels.

SIRIUS offers the subsystems an EDC Integration Tool, a network service to combine distributed EDC's. This tool lets two options for a subsystem to be integrated as an information server into SIRIUS :

- $\quad$ Either the subsystem disposes already of a meta-data-system or it decides to built such a meta-data-system independently within the system or in a specific metainformation-system. In this case the subsystem needs only an additional EDC Local Server in front of his subsystem, which offers the meta-data using the S1-interface to our EDC Integration Tool. In general no modifications will be necessary for the rest of the system.

- If there is no meta-information-system in the local subsystem, the EDC Integration Tool can be used as an interactive comfortable tool to built a local metadata-system. It is important to state that there are only a small set of functions to be implemented and these functions are rather easy tasks. There are several advantages choosing this alternative: 1) integration is possible very quickly and at low cost; 2) the catalogue and class structure presented to the network may be different from the structures used in the local system; 3 ) the local system remains completely untouched.

A SIRIUS-user at one subsystem can use the EDC Visualization Tool (browser) or any self-made tool which is able to access the information using the SIRIUSinterfaces to visualize any local or remote EDC-information. He accesses the network-wide EDC Cat Server which communicates with the Cat Server implementations at other network nodes via SO to communicate the desired informations to the user. Every Cat Server implementation accesses local Metainformations via S1. The Cat Server knows if there is a EDC Local Server in the subsystem or if the meta-information is found in a EDC Local Server in the inetermediate layer (a meta-data-system built-up using the EDC Integration Tool). 
The services offered by a local system can be divided into two categories :

- node independent services : these are services which have to be implemented at every node. These services can be used without any knowledge of the structures and semantics of the information in the subsystem (e.g. "show me all classes of objects in the subsystem", "show me all objects of a specific class", "give me all objects with a specific characteristic", and so on).

- $\quad$ node specific services (additional services) : they use the structure and the semantics of the information and thus cannot be used without any additional knowledge. Furthermore they will need in general specific implementations and thus will provoke modifications in the tools usinf these services.

The functionality offered to the user at any local subsystem can be described as follows.

First the system presents him an overview, which subsystem actually is connected and thus can be accessed, i.e. which subsystem offers a catalogue and a local metaserver. The overview is actually only a kind of navigation help and could be extended to a real navigation layer by different means, e.g. the introduction of a distributed keyword system. In any case the main aspect must be respected that the distributed system is presented to the user logically as a single unit such that the user must not be aware of the distribution.

Using the navigation tool the user can choose a node - in the case of a real navigation layer he wood not be aware of doing so - and request information. The system offers him catalogue and class information from the local database. Internally this is done by a local EDC Server already existing or built up using the EDC Integration tool as described. The user can review catalogue and classes in order to locate information which might be interesting for him. The user then can ask for specific objects which will be sent back to him.

It is possible for the user to navigate from one node to the other. When he changes from node1 to node2 and later again to node1, the data structures of node1 may have been modified considerably resulting in a modified catalogue and classes. The system will offer him the modified information. Thus a decentralized update is possible without any affect outside the local systems.

We summarize the advantages of using SIRIUS as an integration tool :

a) There is a standard abstract object model and a standard protocol which is able to transport any kind of structures information.

b) The dynamic behaviour of the local subsystems is respected, a fact that is very important in the case of environmental information. It is not necessary to exchange clients when the servers change.

c) The local integration is always done using a small set of simple functions.

d) It is possible to start with a small implementation using the interactive tool and the standard browser client to connect to the network (it is even possible to start with no local information system at all if the local information is not yet available electronically).

The SIRIUS-software is based on standard RPC software and C as programming language and thus available on a wide range of systems from personal computers up to powerfull workstations or even minicomputers or mainframes. For a local system every part of SIRIUS might be replaced, e.g. the standard browser, the integration tool or the server stubs may be used but must not be used. SIRIUS includes libraries to program the local system itself (including user interface objects) 
and makes it possible to tailor the local integration scheme to local requirements. Only the standard object protocol is necessary at any node in order to make the network run.

\section{REFERENCES}

DENZER, (1993), Architektur offener Umweltinformationssysteme, Zwischenbericht DFG-Projekt De501/1-1

GÜTTLER, DENZER, (1993),Umweltinformation als verteiltes System, in : A. JAESCHKE et al. (eds), Informatik für den Umweltschutz, 7. Symposium, Ulm, Informatik Aktuell, Springer, pp. 404-416

DENZER, GÜTTLER, (1994a), Integration von Umweltdaten, in B. PAGE et al., Umweltinformatik, Oldenbourg,

DENZER, (1994b), Object-interfaces für Umweltinformationssysteme, in L.M. HILTY et al. (eds), Informatik für den Umweltschutz, 8. Symposium, Hamburg 1994, Metropolis, pp. 343-350

DENZER, GÜTTLER, (1995), Über die Integrationsproblematik bei übergreifenden Umweltinformationssystemen, GI-SI Fachgespräch „Länderübergreifende Umweltinformationssysteme“, Springer 1995 Zürich

SCHIMAK, DENZER, HUMER, KNAPPITSCH, (1994), The Ozone Network for Austria - Technical Concept of a Distributed Environmental Information System, in L.M. HILTY et al. (eds), Informatik für den Umweltschutz, 8. Symposium, Hamburg 1994, Metropolis, pp. 89-96 